\title{
Overexpression of $D E K$ gene is correlated with poor prognosis in hepatocellular carcinoma
}

\author{
HUO-CHUN YI ${ }^{1}$, YA-LI LIU ${ }^{2}$, PAN YOU ${ }^{1}$, JIN-SHUI PAN ${ }^{3}$, JIAN-YAN ZHOU ${ }^{4}$, \\ ZHEN-JIN LIU ${ }^{5}$ and ZHONG-YING ZHANG ${ }^{1}$ \\ ${ }^{1}$ Center of Clinical Laboratory; Departments of ${ }^{2}$ Thoracic Surgery, ${ }^{3}$ Gastroenterology, ${ }^{4}$ Hepatobiliary Surgery and ${ }^{5}$ Pathology, \\ Zhongshan Hospital, Medical College of Xiamen University, \\ Xiamen, Fujian 361004, P.R. China
}

Received February 12, 2014; Accepted September 9, 2014

DOI: $10.3892 / \mathrm{mmr} .2014 .2781$

\begin{abstract}
The oncogene $D E K$ was originally identified as one of the parts of the $D E K-C A N$ fusion gene, arising from the translocation $(6 ; 9)$ in a subtype of acute myeloid leukemia. Since then, $D E K$ has been shown to promote tumorigenesis in a variety of cancer cell types through its roles in inhibiting cell differentiation, senescence and apoptosis. Certain studies have established that $D E K$ is dysregulated in several types of cancer, including hepatocellular carcinoma (HCC). However, its clinical significance in human HCC remains unknown. In this study, the expression of $D E K$ mRNA and protein was examined in 55 surgical HCC specimens and matched non-tumorous tissues. In addition, the correlation between $D E K$ expression and clinicopathological characteristics and prognosis was analyzed. mRNA and protein levels of $D E K$ were found to be significantly overexpressed in the majority of HCC tumors when compared with matched normal hepatic tissues $(\mathrm{P}<0.05)$. In addition, the expression pattern of $D E K$ was closely correlated with differentiation status, portal venous invasion and tumor size $(\mathrm{P}<0.05)$. Kaplan-Meier curves demonstrated that patients with higher $D E K$ expression levels had significantly poorer survival than those with lower $D E K$ expression levels $(\mathrm{P}=0.003)$. In addition, Cox regression analysis demonstrated that the level of $D E K$ expression may be a valuable prognostic factor $(\mathrm{P}<0.05)$. These results suggested that $D E K$ may play a significant role in hepatocyte differentiation and may serve as a useful prognostic marker and biomarker for the staging of HCC.
\end{abstract}

Correspondence to: Dr Huo-Chun Yi or Professor Zhong-Ying Zhang, Center of Clinical Laboratory, Zhongshan Hospital, Medical College of Xiamen University, 201 South Hubin Road, Xiamen, Fujian 361004, P.R. China

E-mail: yhc1818@163.com.cn

E-mail: zzy1123@tom.com.cn

Key words: DEK expression, hepatocellular carcinoma, prognosis, immunohistochemistry, reverse transcription polymerase chain reaction

\section{Introduction}

Hepatocellular carcinoma (HCC) is one of the most common malignancies worldwide. HCC incidence continues to increase, ranking as the second leading cause of cancer-related mortality among males in China (1). Similar to other cancer types, $\mathrm{HCC}$ is characterized by an evident multistage process of tumor progression (2). In spite of the developments in surgical treatment strategies and options made available in recent years, the overall prognosis of HCC patients remains extremely poor, and this is largely due to the high frequency of recurrence or metastasis following surgery (3). Thus, the capacity to subjectively predict the risk of recurrence and subsequent prognosis is essential to guide surgery and chemotherapy.

The oncogene $D E K$ was originally identified as one of the parts of the $D E K-C A N$ fusion gene, arising from the translocation $(6 ; 9)$ in a subtype of acute myeloid leukemia patients (4). The overexpression of $D E K$ has been observed in several malignancies including melanoma, bladder cancer, glioblastoma, retinoblastoma and HCC (5-9). The increasing list of tumor types demonstrating high and easily detectable DEK protein expression indicates the potential of using DEK as a tumor marker (9). A previous study has characterized $D E K$ as a potential urinary and tissue biomarker for transitional cell carcinoma of the bladder of various grades, stages and progression (10). Furthermore, since the amount of $D E K$ expressed in immature cells is greater than that in differentiated cells, it could assist in assessing the differentiation potential of tumor cells (11). Previous studies have identified that $D E K$ overexpression may be linked with the progression of breast cancer, and that $D E K$ may possibly be used not only as a therapeutic target, but also as a breast cancer biomarker for the early diagnosis and prognostic evaluation of breast cancer (12-14).

While $D E K$ overexpression has been observed in $\mathrm{HCC}$, little is known about its clinical significance in this context $(6,15-16)$. In the present study, we examined the expression level of $D E K$ mRNA and protein in HCC surgical specimens and matched normal hepatic tissues, and then analyzed the correlation between $D E K$ expression and clinicopathological characteristics and patient prognosis. 


\section{Materials and methods}

Patients and specimens. Fifty-five pairs of HCC tissues and their adjacent non-cancerous tissues were obtained from patients who had undergone surgical resection at Xiamen Zhongshan Hospital affiliated to Xiamen University, China, between July 2007 and November 2010. The histological diagnoses and tumor grades of all samples were confirmed by an experienced pathologist. The tumor grading was determined based on the Edmondson-Steiner classification (17). Patient characteristics collected for analysis included age, gender, tumor size, Edmondson-Steiner grade, hepatitis history, number of tumor nodules and presence of portal venous invasion. None of the patients engaged in this study received chemotherapy or radiotherapy prior to surgery. Follow-up data was obtained following hepatic resection for all 55 patients. The follow-up period was defined as the interval between the date of the surgery and that of the patient's mortality or the last follow-up. Mortality from other causes was treated as censored cases. This study was authorized by the Research Ethics Committee of Xiamen Zhongshan Hospital affiliated to Xiamen University. Informed consent was obtained from all of the patients. The tissues were collected according to local ethical guidelines and approved beforehand by all participants and the Human Investigation Committee of The Medical College of Xiamen University (Xiamen, China).

All samples were managed anonymously on the basis of ethical and legal standards. For RNA preparation, the resected samples were placed in liquid nitrogen as soon as possible following resection, and stored at $-80^{\circ} \mathrm{C}$. The remaining tissues were used for routine histopathological examination.

Isolation of total RNA and first strand cDNA synthesis. Total RNA was isolated from frozen tumorand matched normal tissues using TRIzol reagent (Invitrogen Life Technologies, Carlsbad, CA, USA) according to the manufacturer's instructions. RNA quality was confirmed by agarose gel electrophoresis. Only RNA without DNA contamination and biodegradable 26S rRNA was prepared for succeeding cDNA synthesis (Fig. 1A). Following photometric quantification, $2 \mu \mathrm{g}$ total RNA was used for a $20 \mu \mathrm{l}$ reverse transcription (RT) reaction containing $4 \mu \mathrm{l}$ X first strand buffer (Promega Corporation, Madison, WI, USA), $2 \mu 10.1 \mathrm{M}$ DTT, $1 \mu \mathrm{l}$ oligo(dT) $)_{15}$ primer $(10 \mathrm{mM})$, $1 \mu \mathrm{d}$ dNTPs $(10 \mathrm{mM})$ and diethylpyrocarbonate-treated water. The reaction mixture was incubated for $1 \mathrm{~h}$ at $42^{\circ} \mathrm{C}$, heated for $5 \mathrm{~min}$ at $95^{\circ} \mathrm{C}$ and placed for $5 \mathrm{~min}$ in an ice bath; the first strand of cDNA was either stored at $4^{\circ} \mathrm{C}$ or used immediately for polymerase chain reaction (PCR).

$P C R$. Primer sequences were as follows: 5'-TCTGTGAGGTTCT

TGATTTGGA-3' (forward) and 5'-CTGTTCCGTTCCTTTTT ACTGC-3' (reverse) for DEK; 5'-ACCTGACCTGCCGTCTA GAA-3' (forward) and 5'-TCCACCACCCTGTTGCTGTA-3' (reverse) for GAPDH. PCR analysis was performed under the following conditions: $4 \mathrm{~min}$ at $94^{\circ} \mathrm{C}, 30$ cycles of denaturation for $20 \mathrm{sec}$ at $94^{\circ} \mathrm{C}$, annealing for $30 \mathrm{sec}$ at $60^{\circ} \mathrm{C}$, extension for $40 \mathrm{sec}$ at $72^{\circ} \mathrm{C}$, and finally $5 \mathrm{~min}$ at $72^{\circ} \mathrm{C}$. The amplification products were analyzed on a $1.5 \%$ agarose gel.
A

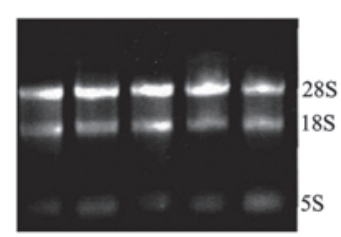

B

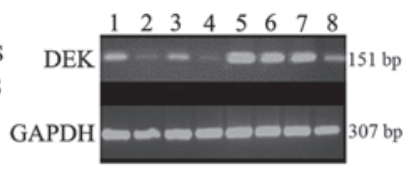

C

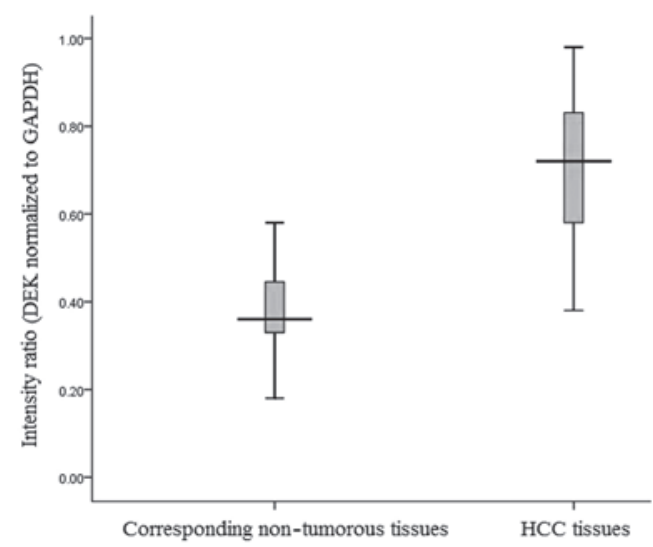

Figure 1. Reverse transcription polymerase chain reaction revealed that the expression levels of $D E K$ mRNA were higher in hepatocellular (HCC) tissues than in corresponding non-tumorous tissues. (A) RNA quality was tested by $1.5 \%$ agarose gel electrophoresis. All extracted total RNA showed integrated $28 \mathrm{~S}$ and $18 \mathrm{~S}$ ribosomal RNA. (B) $D E K$ PCR product amplified from $\mathrm{HCC}$ tissues and adjacent non-tumorous tissues. Lanes 1, 3, 5 and 7 are HCC tissues and lanes 2, 4, 6 and 8 are adjacent tumor tissues. (C) Abundance of $D E K$ mRNA relative to the levels of GAPDH control. $D E K$ mRNA levels in HCC tissues were significantly higher than those in corresponding non-tumorous livers $(0.707 \pm 0.157$ versus $0.391 \pm 0.116 ; \mathrm{P}<0.001)$.

The intensity ratio was the relative expression of $D E K$ normalized to that of GAPDH.

Immunohistochemical staining. A total of 55 HCC tissues and matched normal liver tissues were evaluated by immunohistochemistry. Multiple 5- $\mu \mathrm{m}$ sections were prepared from formalin-fixed, paraffin-embedded tissue blocks. Sections were placed overnight at $4^{\circ} \mathrm{C}$ with mouse anti-human DEK monoclonal antibody (1:100, (Proteintech Group, Inc., Chicago, IL, USA). The streptavidin-biotin peroxidase complex tertiary system (Boster Bio, Pleasanton, CA, USA) was applied in accordance with the manufacturer's specifications. Sections were counter-stained using hematoxylin, dehydrated via gradient alcohol and fixed for observation. All sections were examined independently by two pathologists who were unaware of the patients' clinical status. With regard to the percentage of DEK-positive hepatocytes, immunohistochemical staining was scored as follows: $0,<5 \%$ positive; $1+, 5-25 \%$ positive; $2+, 26-50 \%$ positive; and $3+,>50 \%$ positive. Only nuclear expression was considered to be positive staining. Tissues with moderate to strong nuclear staining were designated as the DEK high expression group (scores $2+$ and 3+). Tissues designated as the DEK low expression group were either devoid of any nucleus staining or scored as 0 or $1+$.

Statistical analysis. SPSS version 16.0 for Windows (SPSS Inc., Chicago, IL, USA) was used for all data analyses. 
A
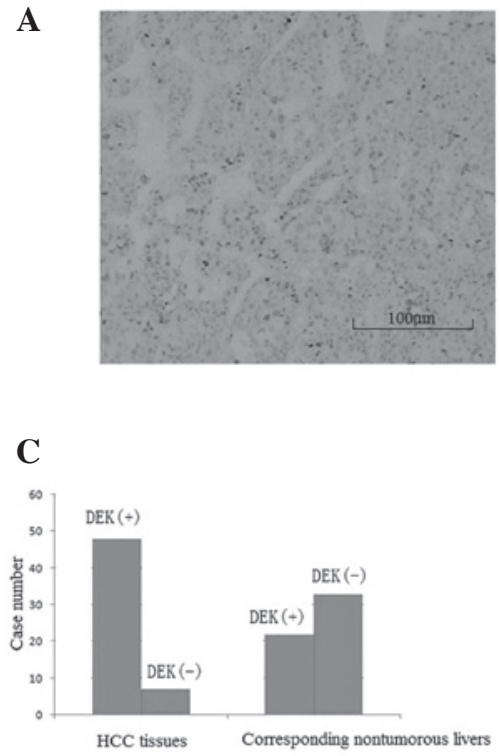

B

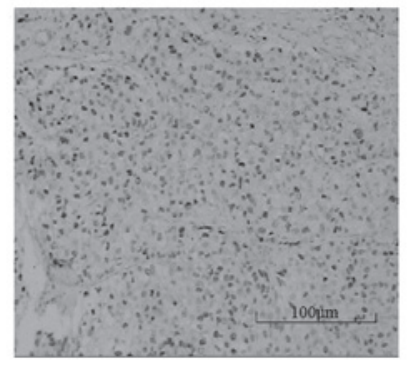

D

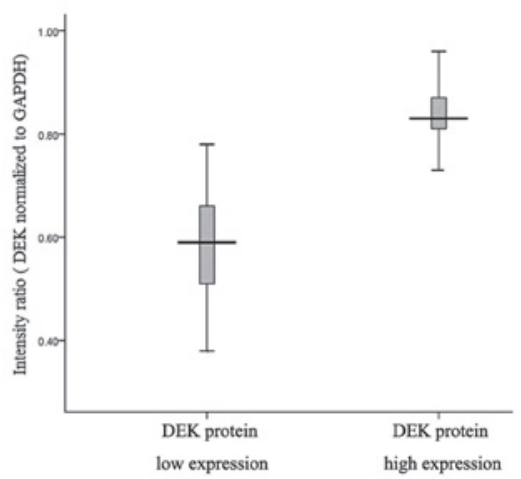

Figure 2. Overexpression of DEK protein in hepatocellular (HCC) tissues compared with corresponding non-tumorous livers. (A) Immunohistochemical detection of the DEK protein moderate expression in HCC tissue (original magnification, x100). (B) Immunohistochemical detection of strong DEK protein expression in HCC tissue (original magnification, x100). (C) The percentage of positive DEK protein expression in HCC tissues was significantly higher than that in corresponding non-tumorous livers [87.3\% (48 of 55) versus 40.0\% (22 of 55); $\mathrm{P}=0.002$ ]. (D) DEK mRNA levels in HCC tissues with high DEK protein expression were significantly higher than in those with low DEK protein expression $(0.841 \pm 0.073$ versus $0.587 \pm 0.106 ; \mathrm{P}<0.001)$.

The paired-samples t-test was used to compare the intensity ratio between $\mathrm{HCC}$ tissues and matching non-cancerous livers. Pearson's $\chi^{2}$ test was used to assess differences in the rate of positive immunostaining between hepatic cancer tissues and adjacent tissues. An independent Student's t-test and Pearson's $\chi^{2}$ test were used to evaluate the correlations between $D E K$ mRNA and protein expression and clinicopathological variables of HCCs, respectively. The Kaplan-Meier method was employed to analyze patient survival, and the differences in survival were evaluated using the log-rank test. The Cox proportional-hazards model was used to confirm factors independently associated with survival. All P-values were based on two-sided statistical analyses, and $\mathrm{P}<0.05$ was considered to indicate a statistically significant difference.

\section{Results}

Significantly increased DEK $m R N A$ and protein expression in HCC tissues. The expression of DEK mRNA was analyzed in $55 \mathrm{HCC}$ samples and matched normal hepatic tissues. Higher expression levels of $D E K$ mRNA were observed in 50 of the HCC tissue samples compared with the matched non-tumor hepatic tissues. DEK mRNA levels in HCC tissues were significantly higher than those in corresponding non-tumorous livers $(0.707 \pm 0.157$ versus $0.391 \pm 0.116 ; \mathrm{t}=18.3$, $\mathrm{P}<0.001$; Fig. 1B and C). By immunohistochemical analysis, the percentage of positive DEK expression in HCC tissues was significantly higher than that in corresponding normal livers [87.3\% (48 of 55) versus $40.0 \%$ (22 of 55); $\mathrm{P}=0.002$; Fig. 2C]. Moreover, the DEK protein levels in HCC tissues were significantly higher than those in the corresponding non-tumorous livers ( $\mathrm{P}<0.01$, Fig. $2 \mathrm{~A}$ and $\mathrm{B})$. Furthermore, an increase in nuclear expression of DEK was observed in HCC tumor tissues, both in intensity and in the positive proportion.

On the basis of the immunohistochemistry data, the $55 \mathrm{HCC}$ cases were divided into the low expression group (score 0 or $1+; n=29$ ) and the high expression group (score $2+$ or $3+; \mathrm{n}=26$ ). The $D E K$ mRNA levels in the DEK high expression group were significantly higher than those in the low expression group $(0.841 \pm 0.073$ versus $0.587 \pm 0.106$; $\mathrm{P}<0.001$; Fig. 2D).

Taken together, these data confirm the overexpression of $D E K$ at the transcription and translation level in human HCC.

Correlation between DEK expression and clinicopathological characteristics. The immunohistochemistry data were analyzed for the correlation of DEK protein expression with clinicopathological features. The expression of DEK protein showed a positive correlation with tumor size $(\mathrm{P}=0.001)$, Edmondson-Steiner grade $(\mathrm{P}=0.025)$ and portal venous invasion $(\mathrm{P}=0.002)$. However, no significant correlation was observed between DEK protein expression and other clinical characteristics, including age $(\mathrm{P}=0.143)$, gender $(\mathrm{P}=0.385)$, hepatitis history $(\mathrm{P}=0.589)$, number of tumor nodules $(\mathrm{P}=0.795)$ and liver cirrhosis $(\mathrm{P}=0.418)$ (Table $\mathrm{I})$. The mRNA levels were compared with the clinical data and found to be associated with the same clinical variables as observed in the protein level analysis.

Follow-up and prognostic value of DEK. The Kaplan-Meier method was used to analyze the correlation of $D E K$ expres- 
Table I. Correlations between DEK protein expression and clinicopathological variables in 55 cases of hepatocellular carcinoma.

DEK expression

Clinicopathological features

$\mathrm{n}$

Low High

$\chi^{2}$

P-value

Gender

Male

43

24

19

0.753

0.385

Female

12

5

7

Age (years)

$<50$

26

11

15

2.148

0.143

$\geq 50$

29

18

11

Tumor size $(\mathrm{cm})$

$\leq 5$

5

10.34

$0.001^{\mathrm{a}}$

$>5$

Hepatitis history

Yes

Edmondson-Steiner grade

3 and 4

Portal venous invasion

Absent

Present

Tumor nodule no.

$\begin{array}{lrrrr}\text { Solitary } & 39 & 21 & 18 & 0.067 \\ \text { Multiple ( } \geq 2) & 16 & 8 & 8 & 0.795 \\ \text { Liver cirrhosis } & & & 4 & 0.656 \\ \text { Absent } & 11 & 7 & 22 & 0.418 \\ \text { Present } & 44 & 22 & \end{array}$

Expression of DEK protein was determined in 55 hepatocellular (HCC) samples by immunohistochemical analysis as described in Materials and methods. Based on the immunohistochemistry data, the 55 cases were divided into a low expression group ( $\operatorname{score} 0$ or $1+; n=29)$ and a high expression group (score $2+$ or $3+; n=26$ ). The correlations between the expression of DEK protein and clinicopathological variables of HCC were evaluated by Pearson's $\chi^{2}$ test. ${ }^{\mathrm{a}} \mathrm{P}<0.05$.

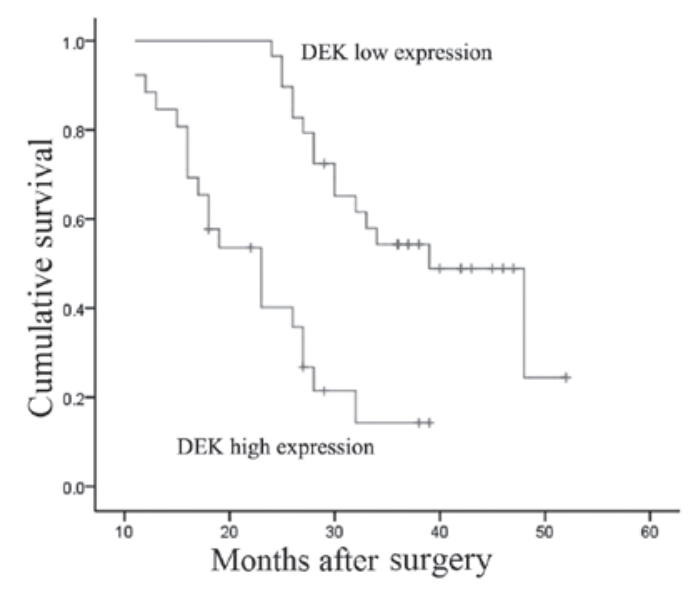

Figure 3. Estimated overall survival according to the expression of DEK protein in 55 cases of hepatocellular carcinoma (HCC) using the Kaplan-Meier method. Based on the results of immunohistochemical staining, the expression of DEK was classified as low expression (0 or $1+; n=29)$ or high expression $(2+$ or $3+; n=26)$. The log-rank test revealed that HCC patients with high DEK expression had a shorter overall survival time than those with low expression $(\mathrm{P}=0.003)$. sion level with the prognosis of HCC patients. The results indicated that the DEK high expression group had a shorter median survival time than the low expression group (23 versus 39 months), and the overall survival rates for the low and high expression groups were significantly different $(\mathrm{P}=0.003$, log-rank test; Fig. 3 ).

To elucidate factors that may predict survival following hepatic resection, univariate and multivariate Cox regression analyses were applied. In the univariate analysis, DEK expression level [hazard risk (HR), 2.273; $\mathrm{P}=0.022)$, Edmondson-Steiner grade $(\mathrm{HR}, 1.542 ; \mathrm{P}=0.039)$ and portal venous invasion $(\mathrm{HR}, 3.145 ; \mathrm{P}=0.015)$ were all significantly associated with survival. However, age, gender, tumor size, hepatitis history, number of tumor nodules and liver cirrhosis were not significantly correlated with survival (Table II). In the multivariate analysis, DEK expression level (HR, 2.974; $\mathrm{P}=0.017$ ), Edmondson-Steiner grade (HR, 2.065; $\mathrm{P}=0.026)$ and portal venous invasion $(\mathrm{HR}, 1.967 ; \mathrm{P}=0.028)$ were identified to be independent prognostic factors of survival (Table II). 
Table II. Multivariate analysis using a Cox proportional-hazards regression model for hepatocellular carcinoma patients.

\begin{tabular}{|c|c|c|c|c|c|}
\hline \multirow[b]{2}{*}{ Clinicopathological variables } & \multirow[b]{2}{*}{$\mathrm{n}$} & \multicolumn{2}{|c|}{ Univariate analysis } & \multicolumn{2}{|c|}{ Multivariate analysis } \\
\hline & & $\operatorname{HR}(95 \% \mathrm{CI})$ & P-value & HR $(95 \% \mathrm{CI})$ & P-value \\
\hline Edmondson-Steiner grade & & & 0.039 & & 0.026 \\
\hline 1 and 2 & 14 & 1 & & 1 & \\
\hline 3 and 4 & 41 & $1.542(1.026-2.746)$ & & $2.065(1.185-2.934)$ & \\
\hline Portal venous invasion & & & 0.015 & & 0.028 \\
\hline Absent & 15 & 1 & & 1 & \\
\hline Present & 40 & $3.145(1.943-5.627)$ & & $1.967(1.173-2.836)$ & \\
\hline DEK & & & 0.022 & & 0.017 \\
\hline Low expression & 29 & 1 & & 1 & \\
\hline High expression & 26 & $2.273(1.474-3.643)$ & & $2.974(1.725-4.139)$ & \\
\hline
\end{tabular}

Insignificant variables with $\mathrm{P}>0.05$ are not listed in the table, including age, gender, tumor size, hepatitis history, number of tumor nodules and liver cirrhosis. HR, hazard risk; CI, confidence interval.

\section{Discussion}

$D E K$ was originally identified in the fusion protein $D E K-C A N$, resulting from the recurrent $\mathrm{t}(6 ; 9)$ translocation in a subset of acute myeloid leukemia patients (4). In addition, previous studies have suggested that $D E K$ is ubiquitously expressed in the majority of mammalian cells (18-19). Subsequently, $D E K$ was reported to be frequently upregulated in aggressive human tumors including melanoma, glioblastoma, retinoblastoma, bladder cancer and HCC (5-8,15-16). DEK has been proven to boost tumorigenesis in numerous cancer cell types through its function of intervening with cell division; inhibiting cell differentiation, senescence and apoptosis (20-22).

In the present study, we measured DEK mRNA and protein expression levels in $55 \mathrm{HCC}$ tissue samples paired with adjacent non-cancerous tissues. The study revealed that levels of $D E K$ mRNA were significantly higher in HCC tissues than in the matching non-cancerous tissues $(\mathrm{P}<0.001)$. Although the present study had a higher number of clinical cases, the result was consistent with that from a previous HCC study, which demonstrated that $D E K$ mRNA levels were higher in 4 of 5 primary HCCs compared with matched non-tumorous liver tissues (13). Lü et al also demonstrated that DEK mRNA levels in HCC tissues were higher than those in paraneoplastic tissues (6).

Furthermore, DEK protein expression was analyzed by immunohistochemistry in the same 55 paired specimens. The percentage of cells with positive DEK expression in HCC tissues was significantly higher than that in corresponding non-tumorous livers (87.3 versus $40.0 \%$; $\mathrm{P}=0.002$ ), comparable with the results from a previous study of DEK protein expression in HCC samples (14). The immunohistochemistry results were in agreement with the mRNA analyses in this study. An increase in nuclear expression of DEK was observed in HCC tumor tissues, both in intensity and in the positive percentage of cells.

In the current study, we divided the HCC cases into low $(n=29)$ and high $(n=26)$ expression groups based on immunohistochemistry scores. $D E K$ mRNA levels in the high expression group were significantly higher than those in the low expression group $(\mathrm{P}<0.001)$. Taken together, these results suggest that overexpression of $D E K$ may be a common event in HCC tumorigenesis.

In this study, the $D E K$ expression data obtained from RT-PCR and immunohistochemistry were analyzed for correlation with clinicopathological features. The results revealed that the expression levels of $D E K$ mRNA and protein were correlated with pathological grade, tumor size and portal venous invasion. Significant differences in the expression levels of $D E K$ mRNA and protein were observed when comparing grade 1 and $2 \mathrm{HCC}$ with grade 3 and 4 HCC. These results are in agreement with a previous HCC study which reported that the level of $D E K$ mRNA was correlated with the histological grade (15). However, Lü et al reported that the difference in expression of $D E K$ mRNA between well- and poorly differentiated HCC was not statistically significant $(\mathrm{P}>0.05)(6)$.

In the present study, the results demonstrated that increased expression of DEK protein was significantly correlated with poor patient outcomes. However, age, gender, tumor size, hepatitis history, number of tumor nodules and liver cirrhosis had no effect on overall survival $(\mathrm{P}>0.05)$, whereas DEK expression, Edmondson-Steiner grade and portal venous invasion were significant predictors of overall survival $(\mathrm{P}<0.05)$. The association of DEK expression with overall survival was consistent with a previous study which demonstrated that increased DEK expression was significantly correlated with poor survival in breast cancer patients (12).

A number of tumorigenic functions of $D E K$ may contribute to the inverse correlation between $D E K$ expression and survival of HCC patients. First, $D E K$ is involved in the inhibition of differentiation and facilitation of cellular transformation (20). Second, $D E K$ inhibits cell apoptosis through its interference with p53 functions (24). Lastly, $D E K$ overexpression has been reported to extend cellular life span, supporting the role of $D E K$ as a senescence inhibitor (25).

In summary, overexpression of $D E K$ in human $\mathrm{HCC}$ is significantly correlated with the prognosis and differentiation potential of HCC, suggesting that $D E K$ may serve as a useful 
prognostic marker. Further studies should be carried out to investigate the precise function and molecular mechanism of $D E K$ in the progression of HCC.

\section{Acknowledgements}

The authors sincerely thank the patients and their families for their cooperation and participation in the study, as well as the staff involved with the study for data collection and management.

\section{References}

1. Murray CJ and Lopez AD: Mortality by cause for eight regions of the world: Global Burden of Disease Study. Lancet 349: 1269-1276, 1997.

2. Sakamoto M: Pathology of early hepatocellular carcinoma. Hepatol Res 37 (Suppl 2): S135-S138, 2007.

3. Tung-Ping Poon R, Fan ST and Wong J: Risk factors, prevention, and management of postoperative recurrence after resection of hepatocellular carcinoma. Ann Surg 232: 10-24, 2000.

4. von Lindern M, Fornerod M, van Baal S, et al: The translocation $(6 ; 9)$, associated with a specific subtype of acute myeloid leukemia, results in the fusion of two genes, dek and can, and the expression of a chimeric, leukemia-specific dek-can mRNA. Mol Cell Biol 12: 1687-1697, 1992.

5. Carro MS, Spiga FM, Quarto M, et al: DEK expression is controlled by E2F and deregulated in diverse tumor types. Cel Cycle 5: 1202-1207, 2006.

6. Lü ZL, Luo DZ and Wen JM: Expression and significance of tumor-related genes in HCC. World J Gastroenterol 11: 3850-3854, 2005.

7. Kappes F, Khodadoust MS, Yu L, et al: DEK expression in melanocytic lesions. Hum Pathol 42: 932-938,2011.

8. Khodadoust MS, Verhaegen M, Kappes F, et al: Melanoma proliferation and chemoresistance controlled by the DEK oncogene. Cancer Res 69: 6405-6413, 2009.

9. Zheng J, Kohler ME, Chen Q, et al: Serum from mice immunized in the context of Treg inhibition identifies DEK as a neuroblastoma tumor antigen. BMC Immunol 8: 4, 2007.

10. Datta A, Adelson ME, Mogilevkin Y, et al: Oncoprotein DEK as a tissue and urinary biomarker for bladder cancer. BMC Cancer 11: 234, 2011.
11. Wise-Draper TM, Morreale RJ, Morris TA, et al: DEK proto-oncogene expression interferes with the normal epithelial differentiation program. Am J Pathol 174: 71-81, 2009.

12. Liu S, Wang X, Sun F, et al: DEK overexpression is correlated with the clinical features of breast cancer. Pathol Int 62: 176-181, 2012.

13. Abba MC, Sun H, Hawkins KA, et al: Breast cancer molecular signatures as determined by SAGE: correlation with lymph node status. Mol Cancer Res 5:881-890, 2007.

14. Privette Vinnedge LM, McClaine R, Wagh PK, et al: The human DEK oncogene stimulates $\beta$-catenin signaling, invasion and mammosphere formation in breast cancer. Oncogene 30: 2741-2752, 2011.

15. Kondoh N, Wakatsuki T, Ryo A, et al: Identification and characterization of genes associated with human hepatocellular carcinogenesis. Cancer Res 59: 4990-4996, 1999.

16. Lin LJ and Chen LT: The role of DEK protein in hepatocellular carcinoma for progression and prognosis. Pak J Med Sci 29: 778-782, 2013

17. Edmondson $\mathrm{H}$ and Steiner P: Primary carcinoma of the liver: a study of 100 cases among 48,900 necropsies. Cancer 7: 462-503, 1954.

18. Sitwala KV, Adams K and Markovitz DM: YY1 and NF-Y binding sites regulate the transcriptional activity of the dek and dek-can promoter. Oncogene 21: 8862-8870, 2002.

19. Hu HG, Scholten I, Gruss C and Knippers R: The distribution of the DEK protein in mammalian chromatin. Biochem Biophys Res Commun 358: 1008-1014, 2007.

20. Grasemann C, Gratias S, Stephan H, et al: Gains and overexpression identify DEK and E2F3 as targets of chromosome 6p gains in retinoblastoma. Oncogene 24: 6441-6449, 2005.

21. Evans AJ, Gallie BL, Jewett MA, et al: Defining a 0.5-mb region of genomic gain on chromosome 6p22 in bladder cancer by quantitative-multiplex polymerase chain reaction. Am J Pathol 164: 285-293, 2004.

22. Castellano G, Torrisi E, Ligresti G, et al: The involvement of the transcription factor Yin Yang 1 in cancer development and progression. Cell Cycle 8: 1367-1372, 2009.

23. Wise-DraperTM,Mintz-Cole RA,Morris TA, etal: Overexpression of the cellular DEK protein promotes epithelial transformation in vitro and in vivo. Cancer Res 69: 1792-1799, 2009.

24. Wise-Draper TM, Allen HV, Jones EE, et al: Apoptosis inhibition by the human DEK oncoprotein involves interference with p53 functions. Mol Cell Biol 26: 7506-7519, 2006.

25. Wise-Draper TM, Allen HV, Thobe MN, et al: The human DEK proto-oncogene is a senescence inhibitor and an upregulated target of high-risk human papillomavirus E7. J Virol 79: 14309-14317, 2005 . 